\title{
Higher braces via formal (non)commutative geometry
}

\author{
Martin Markl
}

\begin{abstract}
We translate the main result of 11 to the language of formal geometry. In this new setting we prove directly that the Koszul resp. Börjeson braces are pullbacks of linear vector fields over the formal automorphism $\varphi(a)=\exp (a)-1$ in the Koszul, resp. $\varphi(a)=a(1-a)^{-1}$ in the Börjeson case. We then argue that both braces are versions of the same object, once materialized in the world of formal commutative geometry, once in the non-commutative one.
\end{abstract}

Mathematics Subject Classification (2010). 13D99, 14A22, 55S20.

Keywords. Koszul braces, Börjeson braces, (non)commutative geometry.

\section{Contents}

Introduction

1. Recollection of formal geometry

1.1. Algebras and coalgebras of symmetric tensors

1.2. Morphisms

1.3. Formal manifolds

1.4. $L_{\infty}$-algebras as homological vector fields

2. The braces

2.1. Koszul braces

2.2. Börjeson braces

References

\section{Introduction}

Let $A$ be a graded commutative associative algebra with a degree +1 differential $\nabla: A \rightarrow A$ which need not be a derivation. Koszul braces are linear

The author was supported by the Eduard Cech Institute P201/12/G028 and RVO: 67985840 . 
degree +1 maps $\Phi_{n}^{\nabla}: A^{\otimes n} \rightarrow A, n \geq 1$, defined by the formulas

$$
\begin{aligned}
\Phi_{1}^{\nabla}(a)= & \nabla(a) \\
\Phi_{2}^{\nabla}\left(a_{1}, a_{2}\right)= & \nabla\left(a_{1} a_{2}\right)-\nabla\left(a_{1}\right) a_{2}-(-1)^{a_{1} a_{2}} \nabla\left(a_{2}\right) a_{1} \\
\Phi_{3}^{\nabla}\left(a_{1}, a_{2}, a_{3}\right)= & \nabla\left(a_{1} a_{2} a_{3}\right)-\nabla\left(a_{1} a_{2}\right) a_{3}-(-1)^{a_{1}\left(a_{2}+a_{3}\right)} \nabla\left(a_{2} a_{3}\right) a_{1} \\
& -(-1)^{a_{3}\left(a_{1}+a_{2}\right)} \nabla\left(a_{3} a_{1}\right) a_{2}+\nabla\left(a_{1}\right) a_{2} a_{3} \\
& +(-1)^{a_{1}\left(a_{2}+a_{3}\right)} \nabla\left(a_{2}\right) a_{3} a_{1}+(-1)^{a_{3}\left(a_{1}+a_{2}\right)} \nabla\left(a_{3}\right) a_{1} a_{2}, \\
& \vdots \\
\Phi_{n}^{\nabla}\left(a_{1}, \ldots, a_{n}\right)= & \sum_{1 \leq i \leq n}(-1)^{n-i} \sum_{\sigma} \varepsilon(\sigma) \nabla\left(a_{\sigma(1)} \cdots a_{\sigma(i)}\right) a_{\sigma(i+1)} \cdots a_{\sigma(n)},
\end{aligned}
$$

for $a, a_{1}, a_{2}, a_{3}, \ldots \in A$. The sum in the last line runs over all $(i, n-i)$ unshuffles $\sigma$, and $\varepsilon(\sigma)=\varepsilon\left(\sigma ; a_{1}, \ldots, a_{n}\right)$ is the Koszul sign (recalled below). As proved for instance in [3], these operations form an $L_{\infty}$-algebra.

The above construction, attributed to Koszul [6], is sometimes called the Koszul hierarchy, cf. also [1, 2, 3, 4, 14. It is, among other things, used to define higher order derivations of commutative associative algebras: the operator $\nabla: A \rightarrow A$ is an order $r$ derivation, $r \geq 1$, if $\Phi_{r+1}^{\nabla}$ vanishes. Higher order derivations are crucial for the author's approach to the BRST complex of the closed string field theory [10, Section 4]; for this reason we believe that the braces might be interesting also for physicists.

The assumption of commutativity of $A$ is crucial. Although the operations $\Phi_{n}^{\nabla}$ make sense for general $A$, they do not form any reasonable structure if $A$ is not commutative.

The noncommutative analog of the Koszul hierarchy was found in April 2013 by Kay Börjeson [5] who also proved that the result is an $A_{\infty}$-algebra; we recall his braces $\left\{b_{n}^{\nabla}\right\}_{n \geq 1}$ in Subsection 2.2. Amazingly, Börjeson's braces are very different from the Koszul ones. While $\Phi_{n}^{\nabla}$ consists of $2^{n}-1$ terms, $b_{n}^{\nabla}$ is for each $n \geq 3$ the sum of 4 terms only!

In 11] we proved that both the Koszul and Börjeson's braces are the twistings of a linear $L_{\infty^{-}}$(resp. $A_{\infty^{-}}$) algebra determined by $\nabla$. In this note we translate this result to the language of formal (non)commutative geometry where $L_{\infty}$ - resp. $A_{\infty}$-algebras appear as homological vector fields. Namely, we show in Theorems 2.1 and 2.8 that both braces are pullbacks of the linear vector field $\nabla$ over a formal automorphism $\varphi$ of the formal graded affine pointed manifold $A$. This automorphism equals

$$
\varphi(a):=\exp (a)-1
$$

in the commutative (Koszul) case, and

$$
\varphi(a):=\frac{a}{1-a}
$$


in the non-commutative (Börjeson) case. An important fact is that the Taylor coefficients of both automorphisms are encapsulated in the same formal sum

$$
\mathbb{1}_{A}+\mu^{[2]}+\mu^{[3]}+\cdots
$$

where $\mu^{[n]}: A^{\otimes n} \rightarrow A, n \geq 2$, is the iterated multiplication in $A$. When interpreted in formal commutative geometry, the associated formal automorphism is (1a) while non-commutative geometry it is (1b). The Koszul resp. Börjeson's braces are thus versions of the same object!

As a pleasant by-product of our calculations, we get an important general formula (23) stated without proof in [11].

Conventions. All algebraic objects will be considered over a field $\mathbb{k}$ of characteristic zero. By $\mathbb{1}_{U}$ or simply by $\mathbb{1}$ when $U$ is understood we denote the identity automorphism of a vector space $U$. We will reserve the symbol $\mu: A \otimes A \rightarrow A$ for the multiplication in an associative algebra $A$. The product $\mu(a, b)$ of elements $a, b \in A$ will usually be abbreviated as $a b$.

A grading will always mean a $\mathbb{Z}$-grading though most of our results easily translate to the $\mathbb{Z}_{2}$-graded setting, i.e. to the super world. To avoid problems with dualization, we will assume that all graded vector spaces are of finite type. For graded vector spaces $U$ and $V$ we denote by $U \otimes V$ their tensor product over $\mathbb{k}$, and by $\operatorname{Lin}(U, V)$ the space of degree $0 \mathbb{k}$-linear maps $U \rightarrow V$.

A permutation $\sigma \in \Sigma_{n}$ and graded variables $\nu_{1}, \ldots, \nu_{n}$ determine the Koszul sign $\varepsilon\left(\sigma ; \nu_{1}, \ldots, \nu_{n}\right) \in\{-1,+1\}$ via the equation

$$
\nu_{1} \wedge \ldots \wedge \nu_{n}=\varepsilon\left(\sigma ; \nu_{1}, \ldots, \nu_{n}\right) \cdot \nu_{\sigma(1)} \wedge \ldots \wedge \nu_{\sigma(n)}
$$

in the free graded commutative associative algebra $\mathbb{S}\left(\nu_{1}, \ldots, \nu_{n}\right)$ generated by $\nu_{1}, \ldots, \nu_{n}$. We usually write $\varepsilon(\sigma)$ instead of $\varepsilon\left(\sigma ; \nu_{1}, \ldots, \nu_{n}\right)$ when the meaning of $\nu_{1}, \ldots, \nu_{n}$ is clear. For integers $u, v \geq 0$, an $(u, v)$-unshuffle is a permutation $\sigma \in \Sigma_{u+v}$ satisfying

$$
\sigma(1)<\cdots<\sigma(u) \text { and } \sigma(u+1)<\cdots<\sigma(u+v) .
$$

\section{Recollection of formal geometry}

In this section we recall basic concepts of formal (commutative) geometry. We start with polynomial algebras, their completions and duals, and explain how they are related to Taylor series of formal maps. We then interpret $L_{\infty}$-algebras as homological vector fields on formal graded pointed affine manifolds. All notions recalled here are standard and appeared in various forms in the literature. Their non-commutative variants are briefly addressed in Subsection 2.2.

\footnotetext{
${ }^{1} \mathrm{~A}$ general case can be controlled by a linear topology, but it would reach beyond the scope of the present paper.
} 


\subsection{Algebras and coalgebras of symmetric tensors}

For a finite dimensional vector space $X$ denote by

$$
\mathbb{S}(X)=\bigoplus_{k \geq 1} \mathbb{S}^{k}(X)
$$

the symmetric (polynomial) algebra generated by $X$. To distinguish the multiplication in $\mathbb{S}(X)$ from other products that may occur in this note we denote the product of two polynomials $p, q \in \mathbb{S}(X)$ by $p \odot q$.

Let $A=X^{*}$ be the linear dual of $X$. One has, for each $n \geq 1$, a nondegenerate pairing

$$
\langle-\mid-\rangle: \mathbb{S}^{n}(X) \otimes \mathbb{S}^{n}(A) \rightarrow \mathbb{k}
$$

given by

$$
\left\langle x_{1} \odot \cdots \odot x_{n} \mid a_{1} \odot \cdots \odot a_{n}\right\rangle:=\sum_{\sigma} x_{1}\left(a_{\sigma(1)}\right) \cdots x_{n}\left(a_{\sigma(n)}\right),
$$

where $x_{1}, \ldots, x_{n} \in X, a_{1}, \ldots, a_{n} \in A$, and the summation ranges over all permutations $\sigma \in \Sigma_{n}$. As a particular case of (3) we get

$$
\left\langle x_{1} \odot \cdots \odot x_{n} \mid a \odot \cdots \odot a\right\rangle:=n ! x_{1}(a) \cdots x_{n}(a) .
$$

Notice the factorial $n$ ! emerging there.

We will need also the completion

$$
\widehat{\mathbb{S}}(X)=\prod_{k} \mathbb{S}^{k}(X)
$$

of $\mathbb{S}(X) ; \widehat{\mathbb{S}}(X)$ is the algebra of power series in $X$. The pairing (3) extends to a non-degenerate pairing

$$
\langle-\mid-\rangle: \widehat{\mathbb{S}}(X) \otimes \mathbb{S}(A) \rightarrow \mathbb{k}
$$

that identifies $\widehat{\mathbb{S}}(X)$ with the linear dual of $\mathbb{S}(A)$. This identification is such that the coalgebra structure on $\mathbb{S}(A)$ induced from the algebra structure of $\widehat{\mathbb{S}}(X)$ is given by the deconcatenation coproduct $\Delta: \mathbb{S}(A) \rightarrow \mathbb{S}(A) \otimes \mathbb{S}(A)$ defined as

$$
\Delta\left(a_{1} \odot \cdots \odot a_{n}\right)=\sum_{1 \leq j \leq n-1} \sum_{\sigma}\left(a_{\sigma(1)} \odot \cdots \odot a_{\sigma(j)}\right) \otimes\left(a_{\sigma(j+1)} \odot \cdots \odot a_{\sigma(n)}\right),
$$

where $a_{1}, \ldots, a_{n} \in A$ and $\sigma$ runs through all $(j, n-j)$ unshuffles. We denote $\mathbb{S}(A)$ viewed as a coalgebra with this coproduct by $\mathbb{S}^{c}(A)$.

The algebra $\widehat{S}(X)$ is the free complete commutative associative algebra generated by $X$. Therefore, for each complete commutative associative algebra $B$ and a linear map $\omega: X \rightarrow B$, there exists a unique morphism $h: \widehat{\Im}(X) \rightarrow B$ of complete algebras such that the diagram

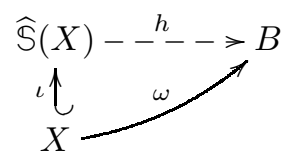


in which $\iota: X \hookrightarrow \widehat{\Im}(X)$ is the obvious inclusion, commutes. In particular, an endomorphism $\phi: \widehat{\mathbb{S}}(X) \rightarrow \widehat{\mathbb{S}}(X)$ is determined by the composition

$$
X \stackrel{\iota}{\hookrightarrow} \widehat{S}(X) \stackrel{\phi}{\longrightarrow} \widehat{\mathbb{S}}(X) \text {. }
$$

Likewise, the coalgebra $\mathbb{S}^{c}(A)$ is the cofree nilpotent cocommutative coassociative coalgebra cogenerated by $A[12$, Definition II.3.72]. This means the following. Let $\pi: \mathbb{S}^{c}(A) \rightarrow A$ be the obvious projection. Then for any nilpotent cocommutative coassociative coalgebra $C$ and for any linear map $\rho: C \rightarrow A$, there exists exactly one coalgebra morphism $g: C \rightarrow \mathbb{S}^{c}(A)$ making the diagram

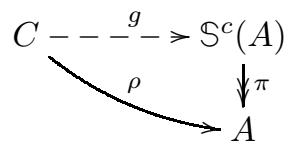

commutative. In particular, any endomorphism $\phi: \mathbb{S}^{c}(A) \rightarrow \mathbb{S}^{c}(A)$ is determined by the composition

$$
\mathbb{S}^{c}(A) \stackrel{\phi}{\longrightarrow} \mathbb{S}^{c}(A) \stackrel{\pi}{\longrightarrow} A .
$$

\subsection{Morphisms}

Let $A$ be a finite dimensional vector space, $A^{*}$ its linear dual, $\mathbb{S}\left(A^{*}\right)$ the symmetric algebra generated by $A^{*}$, and $V$ another vector space. Elements of $\mathbb{S}\left(A^{*}\right) \otimes V$ are polynomials with coefficients in $V$. Each $p \in \mathbb{S}\left(A^{*}\right) \otimes V$ determines a (non-linear) map $f: A \rightarrow V$ as follows.

The pairing (2) in the obvious manner extends to a linear map

$$
\langle-\mid-\rangle: \mathbb{S}^{n}\left(A^{*}\right) \otimes V \otimes \mathbb{S}^{n}(A) \longrightarrow V \text {. }
$$

We associate to every homogeneous polynomial $p_{n} \in \mathbb{S}^{n}\left(A^{*}\right) \otimes V, n \geq 1$, a map $f_{n}: A \rightarrow V$ defined as

$$
f_{n}(a):=\left\langle p_{n} \mid D^{[n]}(a)\right\rangle \in V, a \in A,
$$

where $D^{[n]}: A \rightarrow \mathbb{S}^{n}(A)$ is the 'diagonal' given by

$$
D^{[n]}(a):=\frac{1}{n !}(\underbrace{a \odot \cdots \odot a}_{n \text { times }}) .
$$

The factorial $n$ ! in the definition of $D^{[n]}$ compensates the factorial in (4). Every polynomial $p \in \mathbb{S}\left(A^{*}\right) \otimes V$ is a finite sum

$$
p=p_{1}+p_{2}+p_{3}+\cdots, p_{n} \in \mathbb{S}^{n}\left(A^{*}\right) \otimes V, n \geq 1,
$$

of its homogeneous components. We define $f: A \rightarrow V$ corresponding to $p$ as the finite sum

$$
f=f_{1}+f_{2}+f_{3}+\cdots,
$$

where $f_{n}$ 's are, for $n \geq 1$, as in (7). If the vector space $V$ equals the ground field $\mathbb{k}, \mathbb{S}\left(A^{*}\right) \otimes \mathbb{k}$ is isomorphic to $\mathbb{S}\left(A^{*}\right)$ and we obtain the standard identification of the polynomial ring $\mathbb{S}\left(A^{*}\right)$ with the algebra of regular functions on the affine space $A$. 
We may formally extend the above construction to the space $\widehat{\mathbb{S}}\left(A^{*}\right) \otimes V$ of power series with coefficients in $V$. The sum in (8) corresponding to $p \in \widehat{S}\left(A^{*}\right) \otimes V$ then may be infinite. In this way we interpret power series in $\widehat{\mathbb{S}}\left(A^{*}\right) \otimes V$ as Taylor coefficients of maps $A \rightarrow V .2$

Example 1.1. Let $\mathbb{k}=\mathbb{R}, A$ be the real affine line $\mathbb{R}$ with the basic vector $e:=1 \in A$, and $x \in A^{*}$ be such that $x(e)=1$. For a smooth map $\varphi: A \rightarrow A$ with $\varphi(0)=0$ consider the power series

$$
p:=\varphi^{\prime}(0) x+\frac{\varphi^{\prime \prime}(0)}{2 !} x^{2}+\frac{\varphi^{\prime \prime \prime}(0)}{3 !} x^{3}+\cdots \in \widehat{S}\left(A^{*}\right)
$$

in $x$. One then easily verifies that $p \in \mathbb{S}^{c}\left(A^{*}\right)$ determines, via the above construction, a formal map $f: A \rightarrow A$ given by

$$
f(\alpha e)=\varphi^{\prime}(0) \alpha+\frac{\varphi^{\prime \prime}(0)}{2 !} \alpha^{2}+\frac{\varphi^{\prime \prime \prime}(0)}{3 !} \alpha^{3}+\cdots, \alpha \in \mathbb{R},
$$

i.e. the Taylor expansion at 0 of the smooth $\operatorname{map} \varphi$.

An equivalent representation of formal maps $A \rightarrow V$ is based on the identification

$$
\widehat{\mathbb{S}}\left(A^{*}\right) \otimes V \cong \operatorname{Lin}\left(\mathbb{S}^{c}(A), V\right)
$$

induced by the extended pairing (5). If $q: \mathbb{S}^{c}(A) \rightarrow V$ corresponds, un$\operatorname{der}(10)$, to $p \in \widehat{\mathbb{S}}\left(A^{*}\right) \otimes V$ then $f$ in (8) is, for $n \geq 1$, simply the composition

$$
f=q \circ\left(\mathbb{1}+D^{[2]}+D^{[3]}+\cdots\right) .
$$

Example 1.2. (Continuation of Example 1.1) Let $A$ be again the real affine line $\mathbb{R}$ with the basic vector $e=1 \in \mathbb{R}$. Define $q: \widehat{\mathbb{S}}(A) \rightarrow A$ by

$$
q(\underbrace{e \odot \cdots \odot e}_{n \text { times }}):=\varphi^{(n)}(0),
$$

where $\varphi^{(n)}(0)$ is, for $n \geq 1$, the $n$th derivative of $\varphi$ at 0 . Clearly

$$
q=\varphi^{\prime}(0) \mathbb{1}+\varphi^{\prime \prime}(0) \mu^{[2]}+\varphi^{\prime \prime \prime}(0) \mu^{[3]}+\cdots,
$$

where $\mu^{[n]}: \mathbb{S}^{n}(A) \rightarrow A$ denotes the iterated multiplication in $A=\mathbb{R}$. With this choice, (11) gives the Taylor expansion (9). Notice that (12) does not contain any factorials.

Example 1.3. Suppose that $A$ is a commutative associative algebra and $V=A$. For each $n \geq 1$ one has the map

$$
\mu^{[n]}: \mathbb{S}^{n}(A) \rightarrow A
$$

that takes $a_{1} \odot \cdots \odot a_{n} \in \mathbb{S}^{n}(A)$ to the iterated product $a_{1} \cdots a_{n} \in A$. Consider the morphism

$$
q:=\mathbb{1}+\mu^{[2]}+\mu^{[3]}+\cdots: \mathbb{S}^{c}(A) \rightarrow A .
$$

\footnotetext{
${ }^{2}$ Since we work formally, we do not pay any attention to the convergence issue.
} 
Summation (11) is then the formal map

$$
\exp (a)-1=a+\frac{1}{2 !} a^{2}+\frac{1}{3 !} a^{3}+\cdots, a \in A,
$$

whose inverse clearly equals

$$
\ln (a+1)=\sum_{k \geq 1} \frac{(-1)^{k+1}}{k} a^{k}=a-\frac{a^{2}}{2}+\frac{a^{3}}{3}-\frac{a^{4}}{4}+\cdots .
$$

\subsection{Formal manifolds}

The previous constructions generalize to graded vector spaces. Then $A$ is interpreted as a formal graded pointed affine manifold, ${ }^{3}$ with $\widehat{\mathbb{S}}\left(A^{*}\right)$ playing the rôle of its ring of functions. The power series in $\widehat{\mathbb{S}}\left(A^{*}\right) \otimes V$ represent morphism from $A$ to $V$, where $V$ is interpreted as another formal graded manifold.

Since we work with graded objects, some expressions should acquire signs dictated by the Koszul sign convention. For instance, the terms of the sum in the right hand side of (3) must be multiplied by the Koszul sign $\varepsilon(\rho)$ of the permutation

$$
\rho: x_{1}, \ldots, x_{n}, a_{1}, \ldots, a_{n} \longmapsto x_{1}, a_{\sigma(1)}, \ldots, x_{n}, a_{\sigma(n)} .
$$

This is standard so we will pay no special attention to it. Some issues related to dualization may also arise, but they are not the subject of this note.

Recall that a vector field $\chi$ on a (classical) smooth manifold $M$ is a section of its tangent bundle. The crucial property of vector fields is that the assignment $f \mapsto \chi(f)$ is a derivation of the ring of smooth functions $f: M \rightarrow \mathbb{R}$. There is a contravariant action $\chi \mapsto \varphi^{*} \chi$ of diffeomorphisms $\varphi: M \rightarrow M$ on vector fields characterized by the formula

$$
\varphi^{*} \chi(f)(\varphi(a))=\chi(f \circ \varphi)(a)
$$

in which $f: M \rightarrow \mathbb{R}$ is a smooth function and $a \in M$ a point. We call $\varphi^{*} \chi$ the pullback of the vector field $\chi$ over the diffeomorphism $\varphi$. Let us translate the pullbacks to the situation when we consider instead of $M$ a formal affine graded manifold $A$ as follows.

Vector fields on $A$ are defined as derivations of the algebra of formal functions on $A$, i.e. derivations $\chi$ of the algebra $\widehat{\mathbb{S}}\left(A^{*}\right)$. An automorphism $\varphi: A \rightarrow A$ is given by a power series $p \in \widehat{\mathbb{S}}\left(A^{*}\right) \otimes A$, conveniently represented using the duality by a map $\bar{\phi}: A^{*} \rightarrow \widehat{\widehat{S}}\left(A^{*}\right)$. The invertibility of $\varphi$ is equivalent to the invertibility of the composition

$$
A^{*} \stackrel{\bar{\phi}}{\longrightarrow} \widehat{S}\left(A^{*}\right) \stackrel{\pi}{\longrightarrow} A^{*} \text {. }
$$

Using the universal property of $\widehat{\mathbb{S}}\left(A^{*}\right)$, we uniquely extend $\bar{\phi}$ to an algebra automorphism $\phi: \widehat{\mathbb{S}}\left(A^{*}\right) \rightarrow \widehat{\mathbb{S}}\left(A^{*}\right)$. If $\chi$ is a vector field on $A$, i.e. a derivation of $\widehat{\mathbb{S}}\left(A^{*}\right)$, the action (16) is translated to the adjunction

$$
\varphi^{*} \chi:=\phi^{-1} \circ \chi \circ \phi .
$$

\footnotetext{
${ }^{3}$ We will sometimes omit 'pointed' or/and 'formal' to simplify the terminology.
} 


\section{4. $L_{\infty}$-algebras as homological vector fields}

Recall that an $L_{\infty}$-algebra, also called sh or strongly homotopy Lie algebra, is a graded vector space $L$ equipped with linear graded antisymmetric maps $\lambda_{n}: L^{\otimes n} \rightarrow L, n \geq 1, \operatorname{deg}\left(\lambda_{n}\right)=2-n$, that satisfy a set of axioms saying that $\lambda_{1}$ is a differential, $\lambda_{2}$ obeys the Jacobi identity up to the homotopy $\lambda_{3}$, etc., see for instance [7] or [8].

It will be convenient for the purposes of this note to work with the version transferred to the desuspension $A:=\downarrow L$. The transferred $\lambda_{k}$ 's have degree +1 , are graded symmetric, and satisfy, for each $a_{1}, \ldots, a_{n} \in A, n \geq 1$, the axiom

$$
\sum_{i+j=n+1} \sum_{\sigma} \varepsilon(\sigma) \lambda_{j}\left(\lambda_{i}\left(a_{\sigma(1)}, \ldots, a_{\sigma(i)}\right), a_{\sigma(i+1)}, \ldots, a_{\sigma(n)}\right)=0
$$

where $\sigma$ runs over $(i, n-i)$-unshuffles. In this guise, $L_{\infty}$-algebra is an object $\mathcal{L}=\left(A, \lambda_{1}, \lambda_{2}, \lambda_{3}, \ldots\right)$ formed by a graded vector space $A$ and degree +1 graded symmetric linear maps $\lambda_{n}: A^{\otimes n} \rightarrow A, n \geq 1$, satisfying (17) for each $n \geq 1$.

Let $\mathbb{S}^{c}(A)$ be, as before, the symmetric coalgebra with the deconcatenation coproduct (6). Thanks to the cofreeness of $\mathbb{S}^{c}(A)$, each coderivation $\varrho$ of $\mathbb{S}^{c}(A)$ is determined by its components $\varrho_{n}:=\pi \circ \varrho \circ \iota_{n}, n \geq 1$, where $\pi: \mathbb{S}^{c}(A) \rightarrow A$ is the projection and $\iota_{n}: \mathbb{S}^{k}(A) \hookrightarrow \mathbb{S}^{c}(A)$ the inclusion. We write $\varrho=\left(\varrho_{1}, \varrho_{2}, \varrho_{3}, \ldots\right)$.

In particular, let $\lambda:=\left(\lambda_{1}, \lambda_{2}, \lambda_{3}, \ldots\right)$ be a degree 1 coderivation of $\mathbb{S}^{c}(A)$ determined by the linear maps $\lambda_{n}$. By a classical result [8, Theorem 2.3], (17) shrinks to a single equation $\lambda^{2}=0$. Thus an $L_{\infty}$-algebra is a degree +1 coderivation of $\mathbb{S}^{c}(A)$ that squares to zero.

The linear dual of $\lambda: \mathbb{S}^{c}(A) \rightarrow \mathbb{S}^{c}(A)$ is a degree -1 derivation $\vartheta$ of the algebra $\widehat{S}\left(A^{*}\right)$ such that $\vartheta^{2}=0$. In the language of formal geometry, $\vartheta$ is a degree -1 vector field on the formal affine manifold $A$ that squares to zero. Such an object is called a homological vector field. This is expressed by

Definition 1.4. $L_{\infty}$-algebras are homological vector fields on formal pointed graded affine manifolds.

Example 1.5. Linear vector fields on the classical affine pointed manifold $\mathbb{R}^{k}$ are those of the form

$$
f_{1} \frac{\partial}{\partial x^{1}}+\cdots+f_{k} \frac{\partial}{\partial x^{k}}
$$

where $f_{1}, \ldots, f_{k}: \mathbb{R}^{k} \rightarrow \mathbb{R}$ are linear functions. The formal analog of linear vector fields are derivations $\chi$ of $\widehat{\mathbb{S}}\left(A^{*}\right)$ such that $\chi\left(A^{*}\right) \subset A^{*}$.

In the dual setting, linear vector fields are represented by coderivations $\nabla$ of $\mathbb{S}^{c}(A)$ for which the composition

$$
\mathbb{S}^{\geq 2}\left(A^{*}\right) \smile \mathbb{S}^{c}\left(A^{*}\right) \stackrel{\chi}{\longrightarrow} \mathbb{S}^{c}\left(A^{*}\right) \stackrel{\pi}{\longrightarrow} A
$$

where $\mathbb{S}^{\geq 2}\left(A^{*}\right)$ is the subspace of $\mathbb{S}^{c}\left(A^{*}\right)$ of polynomials with vanishing linear term, is the zero map. Such a coderivation is determined by its restriction to $A \subset \mathbb{S}^{c}(A)$. This restriction is a linear map $A \rightarrow A$ which we denote $\nabla$ again. 
Example 1.6. An important particular case is a linear homological vector field given by a degree +1 differential $\nabla: A \rightarrow A$, or equivalently, by a 'trivial' $L_{\infty}$-algebra $(A, \nabla, 0,0, \ldots)$.

\section{The braces}

We prove that the Koszul braces, as well as their non-commutative analogs recalled in Subsection 2.2 below, are pullbacks of linear vector fields over specific automorphisms. Throughout this section, $A$ will be a graded associative, in Subsection 2.1 also commutative, algebra, and $\nabla: A \rightarrow A$ a degree +1 differential which is not required be a derivation. In Subsection 2.1 we interpret $\nabla$ as a homological vector field on a (commutative) formal manifold or, equivalently, as a trivial $L_{\infty}$-algebra. In Subsection 2.2 we interpret $\nabla$ as a vector field on a non-commutative formal manifold, or as a trivial $A_{\infty}$-algebra, cf. Examples 1.6 resp. 2.7 below.

\subsection{Koszul braces}

The aim of this subsection is to prove

Theorem 2.1. The Koszul braces recalled in the introduction are given by the pullback of the linear homological field $\nabla$ over the formal diffeomorphism $\exp -1: A \rightarrow A$.

We will study pullbacks of $\nabla$ over more general diffeomorphisms and derive Theorem 2.1 as a particular case. This generality would make the calculations more transparent. As a bonus we obtain formula (23) given without proof in [11]. Consider therefore a formal diffeomorphism

$$
A \ni a \longmapsto \varphi(a)=f_{1} a+\frac{f_{2}}{2 !} a^{2}+\frac{f_{3}}{3 !} a^{3}+\cdots, f_{1}, f_{2}, f_{3} \in \mathbb{k}, f_{1} \neq 0,
$$

having the inverse of the form

$$
A \ni a \longmapsto \psi(a)=g_{1} a+\frac{g_{2}}{2 !} a^{2}+\frac{g_{3}}{3 !} a^{3}+\cdots, g_{1}, g_{2}, g_{3}, \ldots \in \mathbb{k} .
$$

Notice that $g_{1} f_{1}=1$.

As in Example 1.3, one can easily check that $\varphi$ is associated to the map $q: \mathbb{S}^{c}(A) \rightarrow A$ given by

$$
q:=f_{1}+f_{2} \mu^{[2]}+f_{3} \mu^{[3]}+\cdots: \mathbb{S}^{c}(A) \rightarrow A .
$$

Let us specify which map $\bar{\phi}: A^{*} \rightarrow \widehat{\widehat{S}}\left(A^{*}\right)$ corresponds to $q$ under the extended pairing (5).

Since $q$ is an (infinite) sum of $f_{n} \mu^{[n]}$ 's, we determine first which map $\bar{\phi}_{n}: A^{*} \rightarrow \mathbb{S}^{n}\left(A^{*}\right)$ corresponds to $f_{n} \mu^{[n]}: \mathbb{S}^{n}(A) \rightarrow A$. Such a map $\bar{\phi}_{n}$ is characterized by the duality

$$
\left\langle x \mid f_{n} \mu^{[k]}\left(a_{1} \odot \cdots \odot a_{n}\right)\right\rangle=\left\langle\bar{\phi}_{n}(x) \mid a_{1} \odot \cdots \odot a_{n}\right\rangle
$$


which has to hold for any $x \in A^{*}$ and $a_{1}, \ldots, a_{n} \in A$. It is obvious that $\bar{\phi}_{n}$ must equal $f_{n} \Delta^{[n]}$, with $\Delta^{[n]}$ the iterated deconcatenation diagonal (6),

$$
\Delta^{[n]}:=\left(\Delta \otimes \mathbb{1}^{\otimes(n-2)}\right) \circ\left(\Delta \otimes \mathbb{1}^{\otimes(n-3)}\right) \circ \cdots \circ \Delta
$$

where we put by definition $\Delta^{[1]}:=\mathbb{1}_{A^{*}}$. We therefore have by linearity

$$
\bar{\phi}=f_{1}+f_{2} \Delta+f_{3} \Delta^{[3]}+f_{4} \Delta^{[4]}+\cdots .
$$

The formula for the map $\bar{\psi}: A^{*} \rightarrow \mathbb{S}^{c}\left(A^{*}\right)$ associated to the inverse $\psi$ of $\varphi$ is analogous, namely

$$
\bar{\psi}=g_{1}+g_{2} \Delta+g_{3} \Delta^{[3]}+g_{4} \Delta^{[4]}+\cdots .
$$

The map $\bar{\phi}: A^{*} \rightarrow \widehat{\mathbb{S}}\left(A^{*}\right)$ extends to a unique automorphism $\phi$ of $\widehat{\mathbb{S}}\left(A^{*}\right)$ given by

$$
\phi\left(x_{1} \odot \cdots \odot x_{n}\right)=\sum_{i_{1}, \ldots, i_{n} \geq 1} f_{i_{1}} \cdots f_{i_{n}} \nabla^{\left[i_{1}\right]}\left(x_{1}\right) \odot \cdots \odot \nabla^{\left[i_{n}\right]}\left(x_{n}\right),
$$

$x_{1}, \ldots, x_{n} \in A^{*}$. There is a similar obvious formula for the extension of $\bar{\psi}$, but we will not need it.

It is however easier to work in the dual setting when vector fields appear as coderivations of $\mathbb{S}^{c}(A)$ or, equivalently, as linear maps $\mathbb{S}^{c}(A) \rightarrow A$. To see how $\varphi$ acts on vector fields in this setup, we need to co-extend the map $q: \mathbb{S}^{c}(A) \rightarrow A$ in (19) to a coalgebra morphism $\mathbb{S}^{c}(A) \rightarrow \mathbb{S}^{c}(A)$. We denote this co-extension by $\phi$ again, believing this ambiguity will not confuse the reader. It acts on $a_{1} \odot \cdots \odot a_{n} \in \mathbb{S}^{n}(A)$ by

$$
\begin{aligned}
& \phi\left(a_{1} \odot \cdots \odot a_{n}\right)= \\
& \quad=\sum \epsilon(\sigma) \frac{f_{i_{1}} \cdots f_{i_{k}}}{k !}\left(a_{\sigma(1)} \cdots a_{\sigma\left(i_{1}\right)}\right) \odot \cdots \odot\left(a_{\sigma\left(i_{1}+\cdots+i_{k-1}+1\right)} \cdots a_{\sigma(n)}\right),
\end{aligned}
$$

with the sum is taken over all $1 \leq k \leq n$, all $i_{1}, \ldots, i_{k} \geq 1$ such that $i_{1}+\cdots+i_{k}=n$, and all permutations $\sigma \in \Sigma_{n}$ such that

$$
\sigma(1)<\cdots<\sigma\left(i_{1}\right), \ldots, \sigma\left(i_{1}+\cdots+i_{k-1}+1\right)<\cdots<\sigma(n) .
$$

Formula (21) can be obtained either by dualizing (20) or directly, using the rule

$$
\Delta \phi=(\phi \otimes \phi) \Delta
$$

describing the interplay between morphisms of coalgebras and coproducts. A $^{\text {n }}$ The rôle of $k$ ! in (21) is explained in Example 2.2 below.

Convention. To shorten the expressions, we will not write the Koszul signs explicitly as they can always be easily filled in. We will also use the shorter $\phi\left(a_{1}, \cdots, a_{n}\right)$ instead of $\phi\left(a_{1} \odot \cdots \odot a_{n}\right)$, etc.

Example 2.2. Formula (21) for $a, b, c \in A$ gives

$$
\begin{aligned}
& \phi(a, b, c)= \\
& \quad=f_{3}(a b c)+\frac{f_{1} f_{2}}{2 !}(a b \odot c+b c \odot a+c a \odot b+a \odot b c+b \odot c a+c \odot a b)
\end{aligned}
$$

${ }^{4}$ Formula (21) must be well-known, but we were unable to locate a reference. 


$$
\begin{aligned}
& +\frac{f_{1}^{3}}{3 !}(a \odot b \odot c+b \odot c \odot a+c \odot a \odot b+a \odot c \odot b+c \odot b \odot a+b \odot a \odot c) \\
& =f_{3}(a b c)+f_{1} f_{2}(a b \odot c+b c \odot a+c a \odot b)+f_{1}^{3}(a \odot b \odot c) .
\end{aligned}
$$

The factorial in (21) therefore removes the multiplicities, so that each type of a term appears only once.

Example 2.3. Let us compute some initial terms of the composition $\bar{\psi} \nabla \phi$ : $\mathbb{S}^{c}(A) \rightarrow A$. For $a \in A$ we have

$$
\bar{\psi} \nabla \phi(a)=g_{1} f_{1} \nabla(a)=\nabla(a)
$$

where we used that $g_{1} f_{1}=1$. For $a, b \in A$,

$$
\begin{aligned}
\bar{\psi} \nabla \phi(a, b) & =\bar{\psi} \nabla\left(f_{2} a b+f_{1}^{2} a \odot b\right) \\
& =\bar{\psi}\left[f_{2} \nabla(a b)+f_{1}^{2}(\nabla(a) \odot b+a \odot \nabla(b))\right] \\
& =g_{1} f_{2} \nabla(a b)+g_{2} f_{1}^{2}(\nabla(a) b+a \nabla(b)),
\end{aligned}
$$

and, finally, for $a, b, c \in A$ one has

$$
\begin{gathered}
\bar{\psi} \nabla \phi(a, b, c)=\bar{\psi} \nabla\left(f_{3} a b c+f_{2} f_{1}(a b \odot c+b c \odot a+c a \odot b)+f_{1}^{3} a \odot b \odot c\right) \\
=\bar{\psi}\left[f_{3} \nabla(a b c)+f_{2} f_{1}(\nabla(a b) \odot c+\nabla(b c) \odot a+\nabla(c a) \odot b)\right. \\
+f_{2} f_{1}(a b \odot \nabla(c)+b c \odot \nabla(a)+c a \odot \nabla(b)) \\
\left.\quad+f_{1}^{3}(\nabla(a) \odot b \odot c+a \odot \nabla(b) \odot c+a \odot b \odot \nabla(c))\right] \\
=g_{1} f_{3} \nabla(a b c)+g_{2} f_{2} f_{1}(\nabla(a b) c+\nabla(b c) a+\nabla(c a) b) \\
+\left(g_{3} f_{1}^{3}+g_{2} f_{2} f_{1}\right)(\nabla(a) b c+a \nabla(b) c+a b \nabla(c)) .
\end{gathered}
$$

Example 2.4. For $\varphi(a)=\exp (a)-1, \psi=\ln (a+1)$ one has

$$
f_{1}=f_{2}=f_{3}=\cdots=1
$$

and

$$
g_{1}=1, g_{2}=-1, g_{3}=2, \ldots, g_{n}=(-1)^{n-1}(n-1) !
$$

With this particular choice, the calculations of Example 2.3 lead, up to implicit Koszul signs, to the Koszul braces $\Phi_{1}^{\nabla}, \Phi_{2}^{\nabla}$ and $\Phi_{3}^{\nabla}$ recalled in the introduction, i.e.

$$
\bar{\psi} \nabla \phi\left(a_{1} \odot \cdots \odot a_{n}\right)=\Phi_{n}^{\nabla}\left(a_{1} \odot \cdots \odot a_{n}\right)
$$

for $n=1,2,3$.

Let us derive a general formula for $\bar{\psi} \nabla \phi: \mathbb{S}^{c}(A) \rightarrow A$. Using (21), one obtains

$$
\begin{aligned}
\bar{\psi} \nabla & \phi\left(a_{1}, \ldots, a_{n}\right)= \\
& =\bar{\psi} \nabla \sum \frac{f_{i_{1}} \cdots f_{i_{k}}}{k !}\left(a_{\sigma(1)} \cdots a_{\sigma\left(i_{1}\right)}\right) \odot \cdots \odot\left(a_{\sigma\left(i_{1}+\cdots+i_{k-1}+1\right)} \cdots a_{\sigma(n)}\right) \\
& =\bar{\psi} \sum \frac{f_{i_{1}} \cdots f_{i_{k}}}{(k-1) !} \nabla\left(a_{\sigma(1)} \cdots a_{\sigma\left(i_{1}\right)}\right) \odot \cdots \odot\left(a_{\sigma\left(i_{1}+\cdots+i_{k-1}+1\right)} \cdots a_{\sigma(n)}\right)
\end{aligned}
$$




$$
=\sum g_{k} \frac{f_{i_{1}} \cdots f_{i_{k}}}{(k-1) !} \nabla\left(a_{\sigma(1)} \cdots a_{\sigma\left(i_{1}\right)}\right) a_{\sigma\left(i_{1}+1\right)} \cdots a_{\sigma(n)} .
$$

The summations in the above display runs over the same data as in (21). The substitution $i_{1} \mapsto i$ converts the last line into

$$
\bar{\psi} \nabla \phi\left(a_{1}, \cdots, a_{n}\right)=\sum_{1 \leq i \leq n} \sum_{\sigma} c_{r} f_{i} \nabla\left(a_{\sigma(1)}, \ldots, a_{\sigma(i)}\right) a_{\sigma(i+1)} \cdots a_{\sigma(n)}
$$

where $\sigma$ runs over all $(i, n-i)$-unshuffles, $r=n-i+1$, and

$$
c_{r}:=\sum_{k \geq 2} \sum_{\substack{i_{2}+\cdots+i_{k}=r \\ i_{2}, \ldots, i_{k} \geq 1}} g_{k} \frac{f_{i_{2}} \cdots f_{i_{k}}}{(k-1) !} \frac{r !}{i_{2} ! \cdots i_{k} !} .
$$

The integer

$$
\frac{r !}{i_{2} ! \cdots i_{k} !}
$$

is, for $r=\left(i_{2}+\cdots+i_{k}\right)$, the number of permutations $\sigma \in \Sigma_{n}$ as in the sum (21) with fixed values $\sigma(1), \ldots, \sigma\left(i_{1}\right)$.

It is a simple exercise on manipulations with power series that

$$
c_{r}:=\left.\frac{d^{r} \psi^{\prime}(\varphi(a))}{d a^{r}}\right|_{t=0} .
$$

Formula (23) is the one we gave without proof in [11, Section 2.4].

In the situation of Theorem 2.1, $\varphi(a)=\exp (a)-1, \psi(a)=\ln (a+1)$, so $\psi^{\prime}(a)=(1+a)^{-1}$, thus $\psi^{\prime}(\varphi(a))=e^{-a}$, therefore $c_{r}=(-1)^{r}$ for $r \geq 1$. Since $f_{s}=1$ for each $s \geq 1$, formula (23) reproduces the Koszul braces in the introduction as direct inspection shows, i.e. (22) holds for every $n \geq 1$. A combinatorial by-product of our calculations is the equality

$$
\sum_{k \geq 2} \sum_{\substack{i_{2}+\cdots+i_{k}=r \\ i_{2}, \ldots, i_{k} \geq 1}}(-1)^{k-1} \frac{r !}{i_{2} ! \cdots i_{k} !}=(-1)^{r} .
$$

We do not know any elementary proof of this fact.

\subsection{Börjeson braces}

A non-commutative analog of Koszul braces was constructed by K. Börjeson. For a graded associative, not necessarily commutative, algebra $A$ and a degree +1 differential $\nabla: A \rightarrow A$, he defined in [5] linear degree +1 operators $b_{n}^{\nabla}: A^{\otimes n} \rightarrow A$ by

$$
\begin{aligned}
b_{1}^{\nabla}(a)= & \nabla(a), \\
b_{2}^{\nabla}\left(a_{1}, a_{2}\right)= & \nabla\left(a_{1} a_{2}\right)-\nabla\left(a_{1}\right) a_{2}-(-1)^{a_{1}} a_{1} \nabla\left(a_{2}\right), \\
b_{3}^{\nabla}\left(a_{1}, a_{2}, a_{3}\right)= & \nabla\left(a_{1} a_{2} a_{3}\right)-\nabla\left(a_{1} a_{2}\right) a_{3} \\
& -(-1)^{a_{1}} a_{1} \nabla\left(a_{2} a_{3}\right)+(-1)^{a_{1}} a_{1} \nabla\left(a_{2}\right) a_{3}, \\
b_{4}^{\nabla}\left(a_{1}, a_{2}, a_{3}, a_{4}\right)= & \nabla\left(a_{1} a_{2} a_{3} a_{4}\right)-\nabla\left(a_{1} a_{2} a_{3}\right) a_{4} \\
& -(-1)^{a_{1}} a_{1} \nabla\left(a_{2} a_{3} a_{4}\right)+(-1)^{a_{1}} a_{1} \nabla\left(a_{2} a_{3}\right) a_{4},
\end{aligned}
$$




$$
\begin{aligned}
b_{k}^{\nabla}\left(a_{1}, \ldots, a_{k}\right)= & \nabla\left(a_{1} \cdots a_{k}\right)-\nabla\left(a_{1} \cdots a_{k-1}\right) a_{k} \\
& -(-1)^{a_{1}} a_{1} \nabla\left(a_{2} \cdots a_{k}\right)+(-1)^{a_{1}} a_{1} \nabla\left(a_{2} \cdots a_{k-1}\right) a_{k} .
\end{aligned}
$$

for $a, a_{1}, a_{2}, a_{3}, \ldots \in A$. He also proved that these operators form an $A_{\infty}$ algebra. In [11] we showed that Börjeson braces are, as their commutative counterparts, a twisting of $\nabla$ interpreted as a trivial $A_{\infty}$-algebra. In this subsection we put these results into the context of non-commutative geometry.

Everything in fact translates verbatim with only minor modifications from the commutative case analyzed in the previous parts of this note. For a finite-dimensional vector space $A$ we denote by

$$
\mathbb{T}\left(A^{*}\right)=\bigoplus_{k \geq 1} \mathbb{T}^{k}\left(A^{*}\right), \mathbb{T}^{k}\left(A^{*}\right):=\underbrace{A^{*} \otimes \cdots \otimes A^{*}}_{k \text { times }}
$$

the tensor algebra of its dual. If $V$ is another finite-dimensional vector space, then every homogeneous non-commutative polynomial $p_{n} \in \mathbb{T}^{n}\left(A^{*}\right) \otimes V$ for $n \geq 1$ determines a map $f_{n}: A \rightarrow V$ defined as in (7), with the only difference that the 'diagonal'

$$
D^{[n]}(a):=(\underbrace{a \odot \cdots \odot a}_{n \text { times }}),
$$

now does not involve the factorial. Since every $p \in \mathbb{T}\left(A^{*}\right) \otimes V$ is a finite sum of its homogeneous components, we can linearly extend the above construction and assign to each $p$ a map $f: A \rightarrow V$. Passing to the completion

$$
\widehat{\mathbb{T}}\left(A^{*}\right):=\prod_{k \geq 1} \mathbb{T}^{k}\left(A^{*}\right),
$$

we interpret non-commutative power series in $\widehat{\mathbb{T}}\left(A^{*}\right) \otimes V$ as non-commutative Taylor coefficients of maps $A \rightarrow V$. Since we have, by duality, an isomorphism

$$
\widehat{\mathbb{T}}\left(A^{*}\right) \otimes V \cong \operatorname{Lin}\left(\mathbb{T}^{c}(A), V\right)
$$

where $\mathbb{T}^{c}(A)$ is the tensor coalgebra with the deconcatenation coproduct, we may equivalently represent non-commutative Taylor coefficients of maps $f: A \rightarrow V$ by linear morphisms $q: \mathbb{T}^{c}(A) \rightarrow V$. Let us give a non-commutative analog of Example 1.3 .

Example 2.5. Let $A$ be an associative algebra. Then the morphism

$$
q:=\mathbb{1}+\mu^{[2]}+\mu^{[3]}+\cdots: \mathbb{T}^{c}(A) \rightarrow A
$$

represents the non-commutative Taylor series

$$
\frac{a}{1-a}=a+a^{2}+a^{3}+\cdots, a \in A
$$

whose inverse equals

$$
\frac{a}{1+a}=a-a^{2}+a^{3}-\cdots, a \in A
$$


Formal non-commutative differential geometry is build upon the above classical non-graded finite-dimensional affine spaces analogously as explained in Subsection 1.3 for the commutative case. That is, $A$ is now a graded vector space interpreted as a non-commutative formal affine pointed manifold with $\mathbb{T}\left(A^{*}\right)$ its non-commutative ring of regular functions. Given another formal non-commutative affine manifold $V$, the non-commutative power series in $\widehat{\mathbb{T}}\left(A^{*}\right) \otimes V$ represent formal maps $f: A \rightarrow V$. By duality, the same formal maps can equivalently be represented by linear morphisms $q: \mathbb{T}^{c}(A) \rightarrow A$.

Vector fields on a formal non-commutative manifold $A$ are derivations of the complete algebra $\widehat{\mathbb{T}}\left(A^{*}\right)$ or, equivalently, coderivations of the tensor coalgebra $\mathbb{T}^{c}(A)$. As in the commutative case, automorphisms act on vector fields by adjunction.

$A_{\infty}$-algebras are non-commutative versions of $L_{\infty}$-algebras recalled in Subsection 1.4, and their historical precursors 13]. They consist of a graded vector space $U$ together with degree $2-n$ linear maps $m_{n}: U^{\otimes n} \rightarrow U, n \geq 1$, such that $m_{1}$ is a differential, $m_{2}$ is associative up to the homotopy $m_{3}$, etc.

As in the case of $L_{\infty}$-algebras, we transfer the maps $m_{n}: U^{\otimes n} \rightarrow U$ to the desuspension $A:=\downarrow U$. These transferred $m_{n}$ 's are all of degree +1 and satisfy

$$
\sum_{u+v=n+1} \sum_{1 \leq i \leq u} m_{u}\left(\mathbb{1}_{A}^{\otimes i-1} \otimes m_{v} \otimes \mathbb{1}_{A}^{\otimes u-i}\right)=0
$$

for each $n \geq 1$.

Since the tensor coalgebra $\mathbb{T}^{c}(A)$ is the cofree nilpotent coassociative coalgebra cogenerated by $A$, each coderivation $\varrho$ of $\mathbb{T}^{c}(A)$ is uniquely determined by its components $\varrho_{n}: A^{\otimes n} \rightarrow A, n \geq 1$, defined as $\varrho_{n}:=\pi \circ \varrho \circ \iota_{n}$, where $\pi: \mathbb{T}^{c}(A) \rightarrow A$ is the projection and $\iota_{n}: A^{\otimes n} \hookrightarrow \mathbb{T}^{c}(A)$ the obvious inclusion. We express this situation by $\varrho=\left(\varrho_{1}, \varrho_{2}, \varrho_{3}, \ldots\right)$.

One in particular has a degree +1 coderivation $m=\left(m_{1}, m_{2}, m_{3}, \ldots\right)$ of $\mathbb{T}^{c}(A)$ determined by the $A_{\infty}$-algebra above. As in the $L_{\infty}$-case, the system (27) is equivalent to a single equation $m^{2}=0$. In other words, an $A_{\infty}$-algebra is a degree +1 coderivation of the tensor coalgebra $\mathbb{T}^{c}\left(A^{*}\right)$ that squares to zero. Its linear dual $\varpi: \widehat{\mathbb{T}}(A) \rightarrow \widehat{\mathbb{T}}(A)$ is a degree -1 derivation that squares to zero, i.e., a homological vector field. We may therefore give the following analog of Definition 1.4:

Definition 2.6. $A_{\infty}$-algebras are homological vector fields on formal noncommutative graded pointed affine manifolds.

The notion of linear vector fields translate verbatim from the commutative case. Here is an analog of Example 1.6:

Example 2.7. A degree +1 differential $\nabla: A \rightarrow A$ extends to a degree +1 derivation of $\mathbb{T}^{c}(A)$, i.e. to a linear homological vector field on the formal non-commutative affine pointed manifold $A$. In other words, it determines a 'trivial' $A_{\infty}$-algebra $(A, \nabla, 0,0, \ldots)$.

We finally formulate a non-commutative analog of Theorem 2.1: 
Theorem 2.8. The Börjeson braces are given by the pullback of the linear homological field $\nabla$ over the formal diffeomorphism

$$
\frac{a}{1-a}: A \rightarrow A
$$

of the formal non-commutative pointed affine manifold $A$.

The proof of this theorem is simpler than that of Theorem 2.1, since no symmetry enters. We leave it to the reader, as well as the non-commutative analog of (24) as appeared in [11, Section 2.2].

\section{References}

[1] F. Akman. On some generalizations of Batalin-Vilkovisky algebras. J. Pure Appl. Algebra, 120(2):105-141, 1997.

[2] F. Akman and L.M. Ionescu. Higher derived brackets and deformation theory. I. J. Homotopy Relat. Struct., 3(1):385-403, 2008.

[3] J. Alfaro, K. Bering, and P. H. Damgaard. Algebra of higher antibrackets. Nuclear Phys. B, 478(1-2):459-503, 1996.

[4] K. Bering. Non-commutative Batalin-Vilkovisky algebras, homotopy Lie algebras and the Courant bracket. Comm. Math. Phys., 274(2):297-341, 2007.

[5] K. Börjeson. $A_{\infty}$-algebras derived from associative algebras with a nonderivation differential. Preprint arXiv:1304.6231, April 2013.

[6] J.-L. Koszul. Crochet de Schouten-Nijenhuis et cohomologie. Astérisque, (Numero Hors Serie):257-271, 1985. The mathematical heritage of Elie Cartan (Lyon, 1984).

[7] T. Lada and M. Markl. Strongly homotopy Lie algebras. Comm. Algebra, 23(6):2147-2161, 1995.

[8] T. Lada and J.D. Stasheff. Introduction to SH Lie algebras for physicists. Internat. J. Theoret. Phys., 32(7):1087-1103, 1993.

[9] M. Markl. A cohomology theory for $A(m)$-algebras and applications. J. Pure Appl. Algebra, 83(2):141-175, 1992.

[10] M. Markl. Loop homotopy algebras in closed string field theory. Comm. Math. Phys., 221(2):367-384, 2001.

[11] M. Markl. On the origin of higher braces and higher-order derivations. To appear in J. Homotopy Relat. Struct., DOI 10.1007/s40062-014-0079-2.

[12] M. Markl, S. Shnider, and J.D. Stasheff. Operads in algebra, topology and physics, volume 96 of Mathematical Surveys and Monographs. American Mathematical Society, Providence, RI, 2002.

[13] J.D. Stasheff. Homotopy associativity of $H$-spaces. I, II. Trans. Amer. Math. Soc. 108 (1963), 275-292; ibid., 108:293-312, 1963.

[14] T. Voronov. Higher derived brackets and homotopy algebras. J. Pure Appl. Algebra, 202(1-3):133-153, November 2005.

Martin Markl

Mathematical Institute of the Academy, Žitná 25, 11567 Prague 1, Czech Republic 
MFF UK, 18675 Sokolovská 83, Prague 8, Czech Republic e-mail: markl@math.cas.cz 